\title{
An Intelligent Lightweight Market Basket Associative Rule Mining for Smartphone Cloud-Based Application To Ease Banking Transaction
}

\author{
A.A. Ojugo \\ Department of Mathematics/Computer Science \\ Federal University of Petroleum Resources \\ Effurun Delta State, Nigeria \\ E-mails: ojugo.arnold@fupre.edu.ng, arnoldojugo@gmail.com, arnoldojugo@yahoo.com \\ R.E. Yoro \\ Department of Computer Science \\ Delta State Polytechnic \\ Ogwashi-Uku, Delta State, Nigeria \\ E-mail: rumerisky@yahoo.com
}

\begin{abstract}
Advent of the Internet has resulted in a consequent explosion of data and its usage. The volume of data generated on daily basis from the exponential increase in the number of users accessing the internet has skyrocketed. The convergence over the Internet, has made it more arduous and imperative to analyze data, due to its tremendously immense sizes that scale up to petabytes of data. The inherent challenges of extracting useful data from such increase has necessitate studies in this guise to seek computational solutions to this challenge therein. We thus propose the use of lightweight market basket analysis for data mining using a hybrid deep learning associative rule mining heuristic.
\end{abstract}

Keywords: Market basket, associative rule mining ARM, data mining, predictive, descriptive, deep learning, evolutionary,

Aims Research Journal Reference Format:

A.A. Ojugo \& R.E. Yoro (2018): An Intelligent Lightweight Market Basket Associative Rule Mining for Smartphone Cloud-Based Application To Ease Banking Transaction. Advances in Multidisciplinary \& Scientific Research Journal. Vol. 4. No.3, Pp 23-34

\section{INTRODUCTION}

The advent of mobile smartphones and its corresponding services has ushered in the era and the realization of the dream for which "Information at your fingertips anytime and anywhere". Advances in ubiquitous computing, has made more prominent a methodology that provides all users with new add-on, consumption and delivery model to IT-related services that are scalable, reliable, device-independent as well as on-demand. It focuses on reducing the inherent limitations of mobile devices through the use of computing techniques for storage and processing of data (Le Guan; Xu Ke; Meina Song and Junde Song, 2011).

Data mining seeks to extract hidden relationships in data-bank or pools, which are located on remotely accessed storage servers or cloud. A great amount of data ranging from commerce, marketing, surveillance fraud detection, scientific discovery, medicine, geographical data system, ecosystem etc - all requires deep analysis and proper decision making, all of which can be achieved via data mining (Ahmet and Ebru, 2013; Ojugo et al, 2018). Data mining thus, uses database analysis to discover the many hidden relations and useful patterns embedded within a data pool, so as to help organizations and users to better focus on the essential data in warehouses by predicting future occurrences and behaviors as it grants business the opportunity to make informed, proactive decisions (Garima and Gbosh, 2014). It use methods such as anomaly detection, association rule mining, neural networks, sequence and path analysis, clustering and classification amongst others to resolve issues in the field of Big-Data (as not all information are useful to the user). Thus, it has become very necessary to extract only useful data from the huge amount of data available (Manpreet and Shivani, 2016), and this is dependent on the kind of data to be mined (Han and Kamber, 2006). 


\subsection{Definition}

Databases are classified based on many criteria such as data models, data types, applications involved etc - each of which may require its own data mining technique. Data mining systems can also thus, classified accordingly. Classifying data mining by data models will often yield relational, transactional, object-relational and data warehouse mining system. In classifying based on data types handled, it yields spatial, time-series, text, stream data, multimedia data mining system, or a World Wide Web mining system. In classifying based on kinds of knowledge they mine (i.e. based on mining functionalities such as data characterization, discrimination, association and correlation analysis) - it can yield predictive (e.g. classification, regression, time series, prediction etc) and/or descriptive (clustering, summarization, association rules, sequence discovery, outlier and evolution analysis). A comprehensive data mining system aims to give multiple and/or integrated mining functionalities. Ultimately, data mining method is classified into predictive or descriptive. Predictive data mining uses historical data to make inferences about future happenings as it uses observed data to construct model for making presages on unseen future events; Whereas, descriptive data mining aims to locate patterns in the data that offer some details about internal hidden relations as well as characterizing the overall properties of the data and represent it in a meaningful way (Ahmed, Turky and Ayman, 2013).

\subsection{Overview of Market Basket Analysis}

Majority of businesses apperceived, continues to amass large amount of data from their customers over time. Also, with the growth of e-commerce applications, such businesses now have an exponential quantity of data to store. Data Mining (also known as Knowledge Discovery in Databases KDD) helps to spot and determine patterns, correlations and/or anomalies in these databases that can avail to engender precise future decisions (Dhanabhakyam and Punithavalli, 2011). Thus, in analyzing data for market basket analysis, businesses are more interested in knowing the item sets that frequently occur together during purchase. The information acquired can be useful for promotions, cross-selling, identification of customer segments based on buying patterns, adjustment of store layouts (i.e. placing items optimally with respect to each other), and for catalogue design (Zhi-Hang, 2016).

Market basket analysis (MBA) is a data mining methods for analyzing the association of data items and their frequency of occurring together from everyday buying and selling. The rudimental conception here is to find the associated pairs of items in a store from the transactional dataset stored in the database. It can become a a springboard therefore, to promote certain items together, to control stocks more perspicaciously, and to increase the probability of purchasing. Thus, managers can place such items associated at the neighboring shelf as a means to give the business a better chance of profit making (even though marketing control and controlling the order of goods is also needed). Online shopping is a form of market basket whereby; shoppers directly buy goods from a seller in real time, through the use of the Internet. In the same way web-shop, virtual stores, an online shop or e-shop are the physical analogy, of purchasing products in a shopping mall.

\subsection{Associative Rule Mining}

Data mining has become crucial to help extract and identify utilizable information (Jian and Chong, 2010). The purchase sequence provides a description of the changes in a buyer's preferences over time, which is what is infer as concept drift of a customer. Association rule mining is quite an effective scheme for data mining using the knowledge discovery in databases (KDD) dataset which often includes data selection, pre-processing, transformation, data mining and interpretation. The extraction of hidden patterns of predictive information, and transforming it into an understandable structure, is the major goal of data mining algorithms (Nidhi, Nikhilendra and Pankaj, 2016). Association rule describes how often items are purchased together when a shopper passes through a point of sale thereby registering the contents of his market basket. This result in immensely colossal accumulations of basket data, that provide data about items that were sold (and in particular, the combinations of items sold together). Thus, finds unsuspected relationship to aid decision making via creating the frequency of items combined (as datasets) for prediction. Frequent items (dataset) are those that occur at least a given number of times for shoppers (also called, minimum support threshold) in the database. Such rule becomes helpful for paramount decisions like promotions, store layout, product pricing, cross-selling amongst others. Association rule mining simply asserts and recuperates the maximum likelihoods, for any item (or a combination of items) to be purchased (Deepa, 2014).

\subsection{Market Basket Analysis Architecture}

\section{The 1-Click Android Shopping Cloud-Based Application}

The 1-Click Android Shopping Cloud-Based Application is depicted in figure below as an $\boldsymbol{n}$-tier architecture model and application. It yield applications are developed to run only on the android platform, and basically - open source with a 3-tier architecture (that is, a frontend, middleware and backend) for clients/endusers. The frontend user can select a particular mall in the city and see offers available in a specific showroom. The frontend also provides offers of local market, available to users' nearby location. Each registered retailers can thus, upload and remove their own offers and advertisement from the application as need arises. 


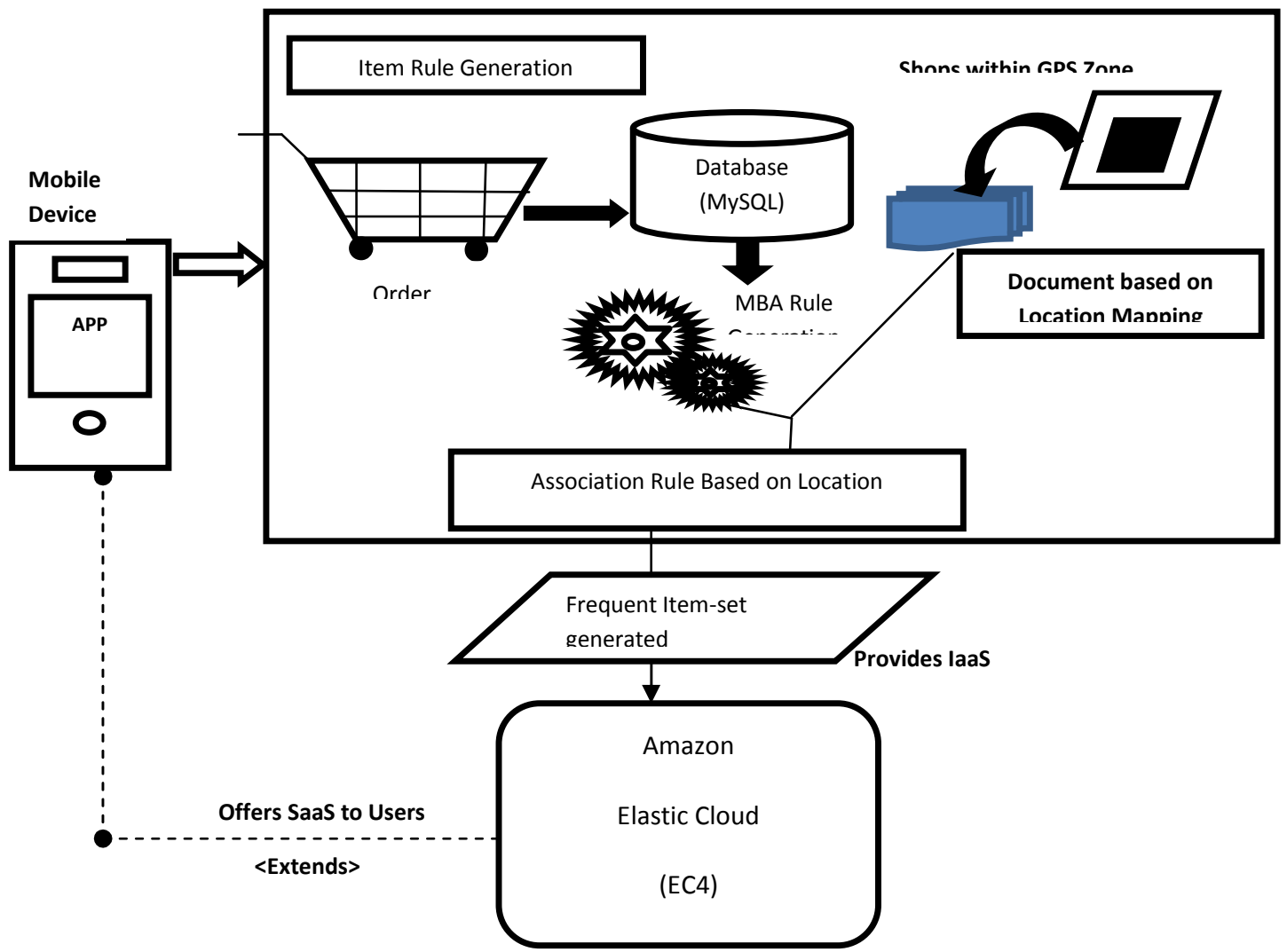

Fig. 1: Market Basket Analysis with Associative Rule Mining Architecture

The middleware tier consists of the web services and houses the Hypertext Transfer Protocol (HTTP), Java Script Object Notation (JSON) and the application. The Web service interfaces between the frontend and backend allowing data exchange for the application. The android application sends its HTTP request, and the web service will query and fetch the requested data from MySQL database located on cloud. The query output is parsed to the frontend via the JSON. The JSON runs in 4-steps as thus: (a) it first allows the application to send a HTTP request using a JSON format to the web service, (b) the query generated by the web services is sent to MySQL database to obtain data from the backend, (c) web service generates a JSON response, sent back to the frontend, and (d) the JSON object needed to decode is displayed as a feedback to the customers in the fourth step. Lastly, at the backend is a web server and MySQL database - to house all previous and recent transactions performed by a user.

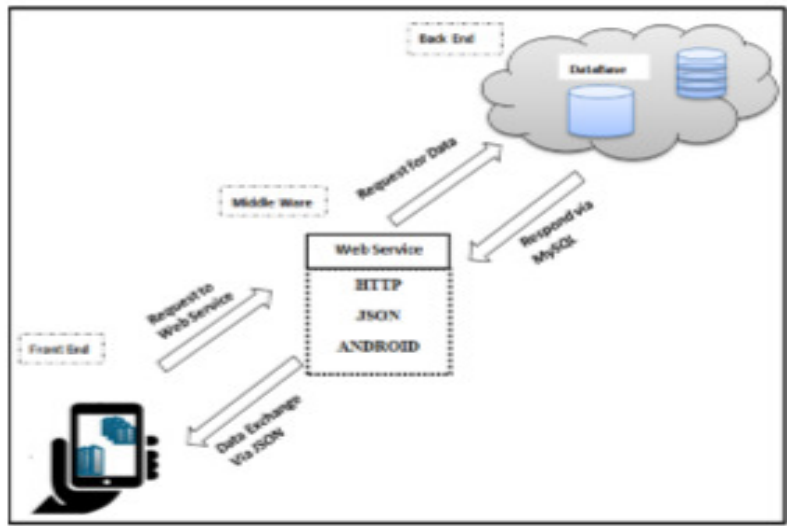

Fig 2: The 1-click Android Application Architecture 
The Agent-Based MBA

The Agent-Based MBA is often a location-based mobile application designed to find readily available services that are nearby as well as associations of the products therein. It can also be formatted as 3-tier framework consisting of a frontend (which is the location-based mobile shopping application of nearby places - and displays association among the purchased products), the middleware provides a web service to generate JSON output from the relational database as well as allows for data exchanges between the mobile application and the servers in cloud, and the backend (using web server and MySQL database) to provide a framework which uses best third-party libraries to avoid unnecessary processes.

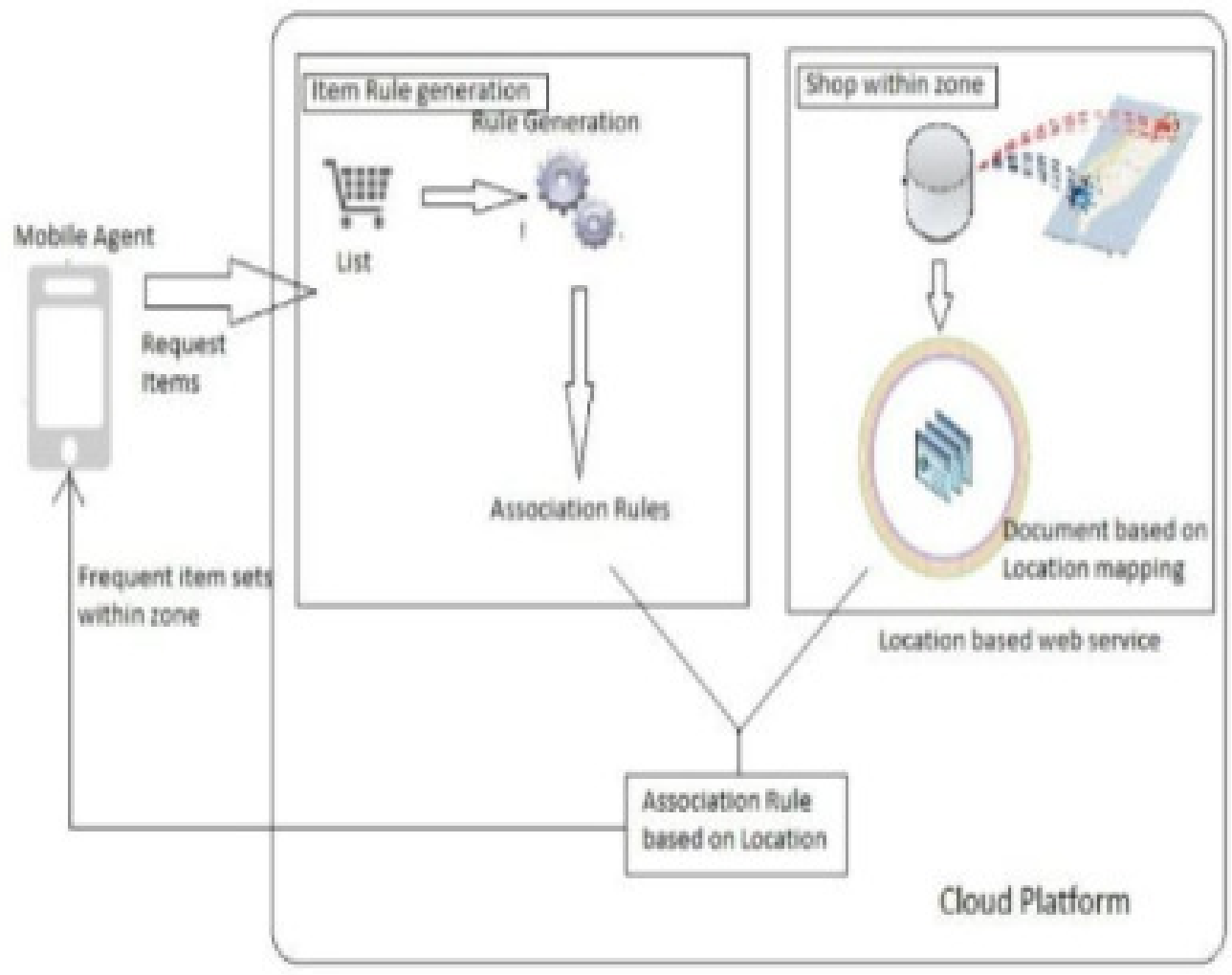

Fig 3: The Mobile Agent-Based MBA Cloud Architecture

The requests are arranged in queue for the confirmation of order by the shopkeeper. Web services are used to manage the connection between front-end and back-end. The framework presented in Fig. 3 is a working principle of this application. Large amount of data collection will be possible using mobile phone as users can place order from anywhere and service provision will be easier. Just few clicks and order will be placed. Also, both customers as well as shopkeepers can take the benefit of mined information for their own benefit and also for marketing the products. 


\subsection{The Mobile Smartphone: Android Platform}

Pernel et al (2013) and Ojugo et al (2015) The Android platform is an eco-system layer of application components that are implemented on a mobile (smartphone) hardware as thus (see fig 4):

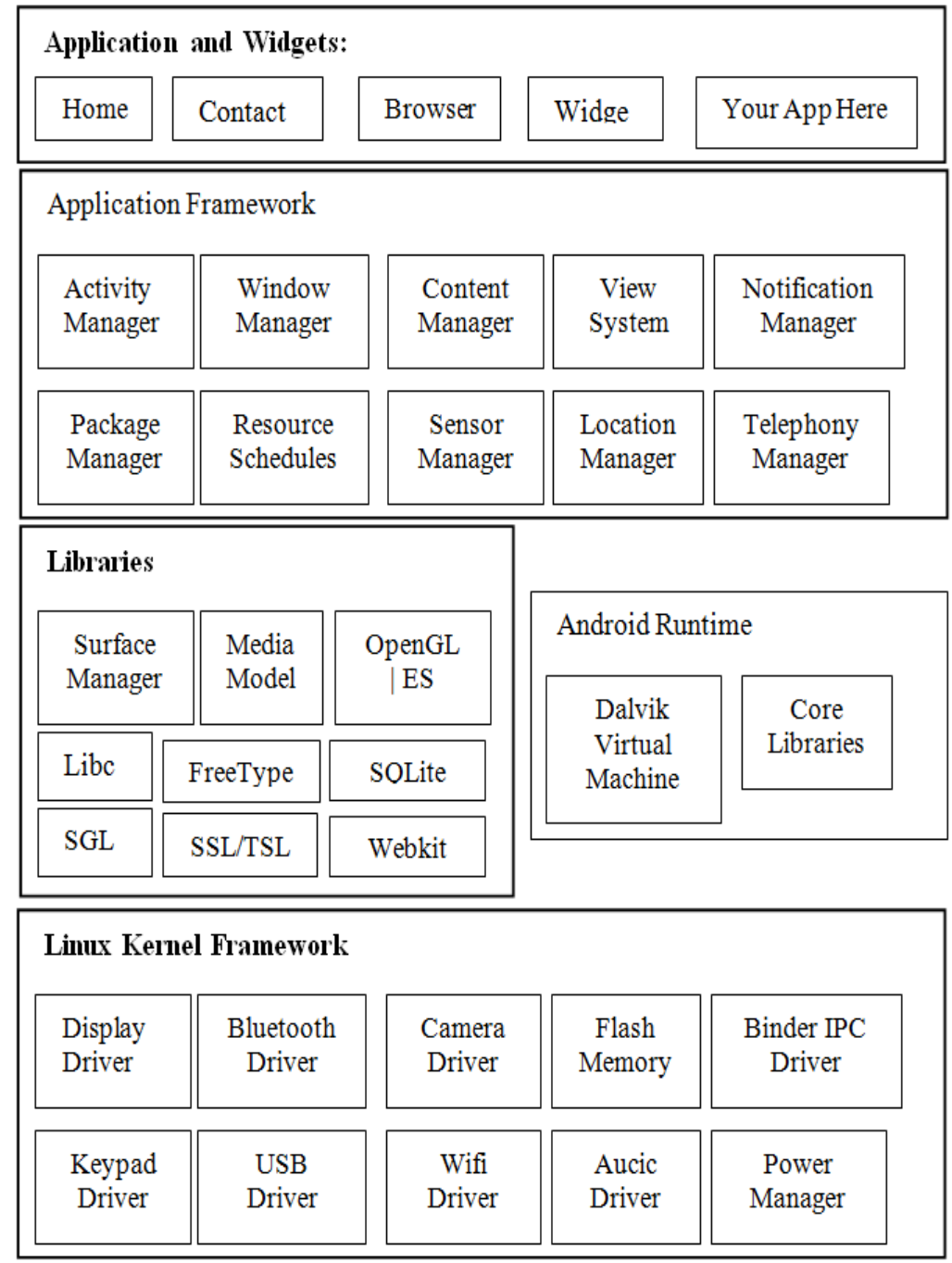

Fig. 4: Android Operating System Platform.

a. Linux OS provides basic functionality such as security, process/memory management and networking to support vast device drivers. It handles human machine interfaces, file systems, network access etc. Its kernel is modified by Google to use low memory killer, specific inter-process communication system, kernel log feats, shared memory system and many other changes as developed. It runs on standard Vanilla Linux, merging specific changes into its kernel. Recent release aimed at real-time Linux kernel is v4.0.3 (Ice Cream Sandwich).

b. Library with Google's libc called Bionic, media/graphics (OpenGL|ES), browser-webkit and light-database SQLite. DVM (Dalvik Virtual Machine) completely differs from Sun's JVM and uses register based byte code to conserves memory, max performance and can instantiate many of its apps multiple times, with each app having its own private copy running. DVM uses Linux for memory management and multi-threading to support the Java language. 
DVM uses bionic (not compatible with glibc) so that its native libraries are faster to implement with small custom pthread to support services such as system and logging capabilities. Writable data segments are small so as to be loaded into memory with each process. This keeps code size small so that Linux loads only once, all readonly pages. Bionic is used: (a) to avoid inclusion of GPL code at user space level in its platform where BSD is used, and (b) for small memory footprint devices with high speed CPUs at relatively low frequencies. Bionic libc does not handle $\mathrm{C}_{++}$exceptions (though omitting such lower level exceptions pose no problem as Java is Android's primary language. It handles exceptions internally). Bionic has no priority inheritance for mutexex as implemented in glibc. Available in its kernel and accessed via own library in system calls, its lack of priority inversion disqualifies it for real-time capability as applied in robotics/automotive. Google's reason for a complete new VM from scratch as accomplished with DVM's register-based byte code is to reduce patent infringement risk. Thus, existing real-time apps modified for JVM cannot easily be ported to DVM.

c. Application Framework provides higher-level services to apps such as Java classes amongst others. Its use can vary between/with varying implementation.

d. Application/Widget are Android routine distributed apps such as email, SMS, calendar, contacts and Web browser.

\section{MATERIALS AND METHOD}

Despite the numerous benefits offered by mobile computing, performing market basket analysis still faces big challenges particularly with the concept of drift and feature evolution.

1. Classifying transactional data pose issues to data mining with concept drift, concept evolution, infinite length and feature evolution. Theoretically, a data stream is infinite in length - making it impractical to store as well as utilize such historical data for training; A naive solution will seeks to ascertain item pairs in each market basket, store assiduously and then run an algorithm finding the most frequent pairs. Such solution however, is impractical as many market baskets containing billions of different item pairs (combination). It is plausible to expect that certain pairs are much more popular than others. Thus, the need to employ stochastic modeling.

2. Previous research on handling such transactional data makes stationary assumption via train/test associative rule mining algorithm on the observed dataset acquired from the same population. However, this deprives the system of the flexibility to adapt to non-existent data and robustness required to handle feature evolution as well as concept drift characteristics inherent in transactional data stream.

3. The ever increasing need and demand for portable device computing continues to necessitate the development of Market Basket Analysis systems for mobile platforms as a major consideration here is the limited speed and memory of portable devices.

Thus, our research objective(s) is to propose a hybrid deep learning associative rule mining model for market basket analysis to resolve the challenges of feature evolution and concept drift; And, thus ensure the effective classification of transactional data stream to handle the issues of big-data.

\section{EXPERIMENTAL FRAMEWORK MODEL}

After a thorough study of all the existing mobile-based cloud models used for designing MBA systems, figure 8 below presents the Architecture of the proposed mobile-based cloud model for MBA showing all its components and how they are interrelated. Proposed model is 3-tier architecture, comprising of a front end, backend and middleware based on the Ojugo and Chiemeke (2018) as in fig. 5.

a. The Front-End contains a cross platform location based mobile application that will display supermarkets within your area using a GPS. The application provided is enable to run on smartphones that runs window, android and iOS mobile Operating Systems. It provides an interface where customers will login to create orders for items. The order created for the current items is then be compared with the previous transactions of same customer in other to be able to generate frequent item sets for recommendations.

b. The Middleware: the middleware component provides a web service interface that helps in data exchange between the frontend and the backend of the model through the use of the server in the cloud. Queries are generated by the frontend users, which is usually in JSON (JavaScript Object Notation) output format, which is then sent to the relational database for response.

c. The Backend: at the backend provides an interface where computing resource such as memory and processing are made available through the help of the Amazon Elastic Cloud (EC4). EC4 houses both the relational database and the Apache tomcat Server that helps the entire model to function at an optimum level. The EC4 provide an Infrastructure-as-a-service platform for hosting our resources. 
Client data stored in smartphones have become easy targets for malicious attempts. Clients may not understand security features as provided in varying forms, needed to be in place as they constantly access Internet and other computing platforms. Thus, study proposes a reliable modeled framework that seeks to help users uncover and detect socially engineered attacks that are aimed at smartphones.

The framework seeks to bring a sense of control to many of the Service providers (infrastructure and services) as well as grant a failsafe to mobile smartphone via a single platform via mobile computing. It is an extension of the miniaturization process and faster computing on Moore's law, bringing about dependable and secure data storage capability to users via portable mobile devices. The framework is designed to thwart efforts geared towards social engineering attacks via phishing, vishing, smishing and whaling (to mention a few). This is because many transactions are also bank-related. It is now customary that very often, most clients use digipass (or tokens) to authenticate their every mobile banking transactions or messages (between them and their banking institutions) from their trusted device (smartphone) via either the hardware or software form for message and transaction authentication as well as client authorization. As thus:

a. Cryptogram - [6] GSM and CDM/FDM are grouped under second-generation systems. GSMs are developed to resolve incompatibilities in the cellular technologies so that a number of subscriber units can be used. Its basic feats are: subscriber Identity Module (SIM) portable smartcard-like plug-in device that stores a user's identification number, the network a client is authorized to use, encryption keys, and other info specific to a user or subscriber. Terminals are generic until SIM is inserted so that the subscriber unit and SIM roams; Transmissions between base transceiver and user is encrypted with $\boldsymbol{A} \boldsymbol{5}$ cipher; while, $\boldsymbol{A} \boldsymbol{3}$ cipher helps authenticate calls making it private to a user. GSMs support data and image services based on ISDN (Integrated Service Digital Network) model with user data rates upto 9.6kbps.

The advent of third-generation communication system allowed mobile subscribers and personal communication services to incorporate set of standards and services unto the mobile unit (making them smartphones) so that it can then support data in form of voice, files, image and video. Though, housed within the device is the cipher (A5 and A3) that helps to authenticate data sent between devices and the service provider. However, banks can provision for extra layer of cryptogram that allows further ciphering so that the data packets (messages or transaction) initiated by a client as sent via a client-trusted device encoded for adequate authentication by the bank. The cryptogram contains all transaction data, including: registered device in use, transaction amount, and recipient account details.

b. Digipass (Hard/Soft tokens) addresses clients' transaction authorization and message authentication weakness via and by assuring the bank that only a registered/authorized client can authorize a legitimate transaction, so long the bank is able to verify the user details which includes (not limited to): registered device in use, account details and benefactors (recipient from another bank) etc [14-16].

c. Data Configurable Model allows for trusted computing via virtualization. Virtualization simply is the process of decoupling hardware from an OS on a physical machine. The Internet (and Financial institutions today), via mobile banking provide users with multiple isolated environ or sites, known as virtual machines (VMs) on a single host. VM is a virtualized representation of a physical machine that runs or is maintained on a host machine by software virtual machine monitor (hypervisor), to provide trusted computing, a mechanism that allows financial institutions to verify their security posture through hardware/software controls. A key component is the trusted platform module (TPM), which is a cryptographic component that provides a root of trust for building such a trusted computing base. Its goal is to move cryptographic computations into a locked virtual area, which is not under control of entities on the host platform. TPM works only in non-virtualized environs. A virtual Trusted Platform Module is provided according to standard specs by creating an instance of TPM for each VM on a trusted platform. All these layers of security - helps to act as measures to ward-off hackers and intruders [17-20].

d. Web Services API - Framework seeks to implement an AES-256 encryption, which is easily ported and supported by SSL/TSL. We adopt this for: (a) it is computationally secure against brute-force, (b) flexible, (c) small-size Java codes allows support for C-language, (d) memory size required is small as ported on Android platform as it has no effect on the performance of memory and speed of the smartphone, and (e) ease of integration as implemented with Java and support for C-language into its web browsers with ease of connectivity. Also, the Chrome and Firefox Web-browsers can be used as they all allow AES-256 encryption. Since, data transfer over the Internet between a client and his/her service provider remains unprotected, no matter how good SSL in use is. 


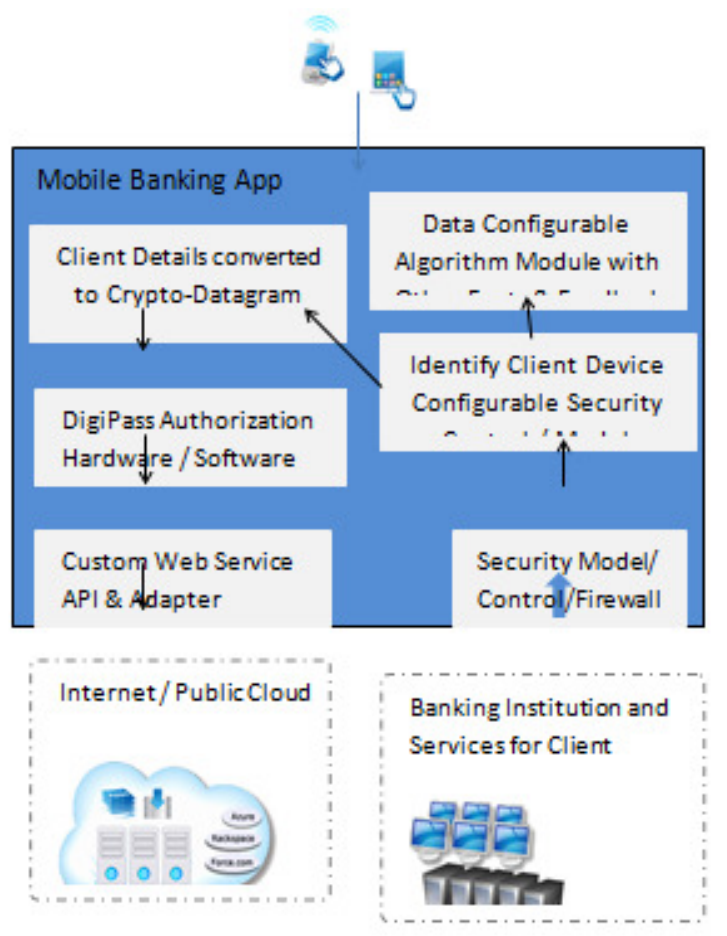

Fig 5: Application and Data Model For Smartphone

The study provides a framework security model for clients using smartphones, and operable on the Android v2.4 platform with support for web-service to allow easy access to Internet connectivity and connection to remote server/cloud-services via API call (adapter). Framework masks all technical nuances between application-model and data-models such as session management, connectivity-issues, transaction authentication and message authorization between a client and his/her bank. Its security is first handled via the digipass that generates a user personal identification number (PIN) from the hardware device (called a token). It is worthy to note that the device is already registered to the user so that whatever token generated can allow the user to log unto his account via the A5 and A3 ciphers provided natively by the mobile smartphone device. Furthermore, the client's smartphone device is then used to log unto the bank's platform bypassing their firewall - having been granted access via generated token so that sent message are authenticated and initiated transaction are verified by the bank to originate from an authorized client.

The system then uses the cryptogram to ensure that transactions and messages are properly formatted according to the bank so that they are allowed to efficiently be transmitted via the AES-256 crypto-system on SSL/TSL as applied to all data. Its client end-to-end encryption solution uses AES-256 to protect its data; while SSL protects username and password (see fig. 1). Tools used for the development of this native app include Android SDK, Apache XAMPP and Google's Android Studio. This native app is enabled and ported on any Android platform from v2.2 with forward compatibility.

The proposed framework was tested through comparison and evaluation with live and running scenario of a community-cloud provider (as well as connected over the Internet) and a user. The user is natively connected to the Internet via his/her smartphone device, and also subscribed for laaS services via FUPRE community-cloud to USbased cloud service provider for purpose of installing some proprietary mobile smartphone applications. Product details for the cloud provider, options available, their corresponding values yielding provider's level of Client Trusted Process Model of proposed model is as seen in Table 1. 
Table 1: Smartphone Internet Provider's Product Options

\begin{tabular}{|l|l|}
\hline Product Details & Options set \\
\hline Registration Date: & $5 / 27 / 2012$ \\
\hline Product/Service: & Online Traders - VPS Value Edition \\
\hline CNS Subscription ID: & 118223 \\
\hline VM: & VM118223.tradersvps.net \\
\hline IPv4 Address: & 173.228 .134 .65 \\
\hline Number of Snapshots: & 1 \\
\hline CPU Cores: & 2 \\
\hline RAM (MB): & 640 \\
\hline DISK (GB): & 20 \\
\hline Two-factor Authentication: & No \\
\hline VNC: & Do not install VNC \\
\hline Operating System OS: & Traders VPS Windows 2003 (x86) Enterprise Edition R2 \\
\hline Language: & English \\
\hline Datacenter: & NYC \\
\hline Payment Method: & MasterCard, Visa \& Am- Express \\
\hline First Payment Amount: & $\$ 30.00$ USD \\
\hline Recurring Amount: & $\$ 30.00$ USD \\
\hline Next Due Date: & $8 / 27 / 2016$ \\
\hline Billing Cycle: & Monthly \\
\hline Status: & Active \\
\hline
\end{tabular}

The location of the datacenter, the description of the Virtual Machine, monthly rate paid by the user, the security control provided by the provider and other product information are shown in Table 1. It is seen from Table 1 that the only security control provided by provider is "Two-factor Authentication". Security control with documentation for proposed framework using simulation is shown in Table 4. Proposed framework uses 3-security control systems at point of data configurable algorithms. The user trust level increases with the number of operational and well document security controls. Comparing the initial values with Table 4, the proposed framework is capable of increasing the trust level of the client by about $72 \%$ when compared to the existing cloud system. The users trust can increase above this value as more appropriate security controls are put in place to clear the user's doubt and enhance the trust level.

\section{CONCLUSION}

ARM plays an important role in Market Basket Analysis by finding the frequency of occurrence of items in a transactional dataset. Its use aims to maximize the social relationship with users/clients. With cloud computing, mobile processing and storage is transferred to cloud as a server - helping to resolve challenges such as low power output, low memory, improve performance and speed inherent with mobile devices. It can be deduced based on reviewed literature that previous studies did not consider the benefit of using larger amount of dataset to perform ARM. Also, the problem inherent with transactional dataset for mobile/cloud computing providing ready-made solution, were not considered. The study designed a model for MBA using ARM for smartphones that will run on the amazon elastic cloud (EC4). System seeks to integrate 3-tier model architecture of frontend, middleware and backend. Once the application is developed it will be able to generate recommendations to customers on particular supermarket products to purchase on their next visit to the online shopping mall. Also, the use of the location based services which integrate the GPS will gives flexibility and attractive looks to the application. Furthermore, the application when developed will provide a Software-as-a-Service platform to the mobile users to get their recommendations for items. 


\section{REFERENCES}

[1] Abolfazli, S., Sanaei, Z., Ahmed, E., Gani, A., \& Buyya, R. (2013). Cloud-Based Augmentation for Mobile Devices: Motivation, Taxonomies, and Open Challenges. IEEE Communications Surveys \& Tutorials, 1-32. doi:10.1109/SURV.2013.070813.00285

[2] Abolfazli, S., Sanaei, Z., Gani, A., Xia, F., \& Yang, L. T. (2013). Rich Mobile Applications: Genesis, taxonomy, and open issues. Journal of Network and Computer Applications, 345-362.

[3] Agrawal, R., \& Srikant, R. (1994). Fast algorithms for mining association rules in large databases., . Proceedings of the 20th International Conference on Very Large Data Bases, VLDB, (pp. 487-499). Santiago. Retrieved January 23, 2017

[4] Agrawal, R., Imielinski, R., \& Swami, \&. (1993). A Mining associations Between Sets of Items in Massive Databases. Page 207-216. Chicago, Washington DC, USA: In Proceedings of the ACM SIGMOD Conference on Management of Data. Retrieved September 24, 2016

[5] Angoss. (2012, July 7). Market Basket Analysi. Toronto, Canada: A.S Cooperation.

[6] Annie, L., \& Kumar, A. (2012). Market Basket Analysis for a Supermaket Based on Frequent item Mining. International Journal of Computer Science, IX(5), 34-43. Retrieved January 10, 2017

[7] Awodele, O., Izang, A. A., Kuyoro, S., \& Osisanwo, F. (2016). Big Data and Cloud Computing Issues. International Journal of Computer Applications, 133(12), 0975-8887.

[8] Borgelt, C. (2014). Find Frequent Item Sets and Association Rules with the Apriori Algorithm. Retrieved from C. Borgelt's home page : http://www.borgelt.net/doc/apriori/apriori.html

[9] Deshmukh, H., ANKIT, R., LALIT, V., DHIRAJ, G., \& RAHIL, K. (2016). Android Application for Searching and Shopping Based on Cloud. INTERNATIONAL JOURNAL OF PURE AND APPLIED RESEARCH IN ENGINEERING AND TECHNOLOGY, 4(9), 1368-1374.

[10] Dhanabhakyam, M., \& Punithavalli, M. (2011, July). A Survey on Data Mining Algorithm for Market Basket Analysis. Global Journal of Computer Science and Technology, XI(11), 13-21. Retrieved Feburary 8, 2017

[11] Dhar, S. (2012). From Outsourcing to Cloud Computing: Evolution of it Services. Management Research Review, 35(8), 664-675.

[12] Fangming, L., Peng, S., Hai, J., Linjie, D., Jie, Y., Di, N., \& Bo, L. (2013). Gearing Resource-Poor Mobile Devices with Powerful Clouds: Architecture, Challenges and Applications. IEEE Wireless Communications Magazine, Special Issue on Mobile Cloud Computing, 20(3), 14-22.

[13] Jason, B. (2013). A Tour of Machine Learning Algorithms. Journal of Intelligent Learning Systems and Applications, 9(4), 50-62.

[14] Kamalraj, R., Kannan, A., Vaishnavi, S., \& Suganya, V. (2012). A Data Mining Based Approach for Introducing Products in SaaS ( Software as a Service). International Journal Of Engineering Innovation \& Research, I(2), 210-214.

[15] Khan, A. u., Othman, M., Madani, S. A., \& Khan, S. U. (2014). A Survey of Mobile Cloud Computing Application Models. IEEE Communications Surveys Tutorials, 16(1), 393-413. doi:10.1109/SURV.2013.062613.00160

[16] Lanois, P. (2010). Caught in the Clouds: The Web 2.0 Cloud Computing and Privacy. Northwestern University School of Law Northwestern Journal of Technology and Intellectual Property, IX(2), 44-52.

[17] Maheshwari, N., Pandey, N. K., \& Agarwal, P. (2016). Market Basket Analysis using Association Rule Learning. International Journal of Computer Applications , 0975-8887. Retrieved Feburary 12, 2017, from www.ijcaonline.org

[18] Marafi, S. (2014, March 19). Market Basket Analysis with R. Retrieved October 16, 2016, from Marafi Salem Blog : http://www.salemmarafi.com/code/market-basket-analysis-with-r/

[19] Meherwar, F., \& Maruf, P. (2017). Survey of Machine Learning Algorithms for Disease Diagnostic. Journal of Intelligent Learning Systems and Applications, 1-16. Retrieved from http://www.scirp.org/journal/jilsa

[20] Mell, P., \& Grance, T. (2011, september). The NIST Defination of Cloud Computing. Gaithersburg, MD, United States. Retrieved September 2016, from http://csrc.nist.gov/publications/nistpubs/800-145/SP800-145.pdf

[21] Ogu, E. C., Alao, O., Omotunde, A., Ogbonna, A., \& Izang, A. (2014). Partitioning of Resource Provisions for Cloud Computing Infrastructure against DoS and DDoS Attacks. International Journal of Advanced Research in Computer Science, $V(7), 67-71$. doi:10.13140/2.1.2259.7129

[22] Ojugo, A.A and Chiemeke, S.C., (2018). Inventory management and prediction using market basket analysis associative rule mining: a memetic algorithm-based approach. Accepted Technical Report by the Nigerian Computer Society Journal of Computer Applications.

[23] Patrick, T., Laduzenski, S., Edwards, D., Ellis, N., Boezaart, A. P., \& Aygtug, H. (2011). Use of machine learning theory to predict the need for femoral nerve block following ACL repair. Pain Medicine, 12(10), 1566-1575. 
[24] Robin, D. (2010). MACHINE LEARNING OVERVIEW. Retrieved from Intelligence World of Computing.: http://intelligence.worldofcomputing.net/machine-learning/machine-learning-overview.html\#.WcpQ4GhSzlU

[25] Rosaria, S. (2015). How to build a Market Basket Analysis Engine. Retrieved from Analyticbridge: http://www.analyticbridge.datasciencecentral.com/profiles/blogs/how-to-build-a-market-basket-analysis-engine

[26] Ruxandra-Stefania, P. (2012). Data Mining in Cloud Computing. Database Systems Journal, III(3), 67-71.

[27] Shivani, R., Rajeshree, P., Laxman, E., \& Yogesh, A. (2015). One Click Andriod Application for Shopping Based on Cloud. International Journal of Scientific Research in Science, Engineering and Technology, I(5), 192-196.

[28] Sumaira, S. (2010). Mobile Cloud Computing Architecture Applications and Services. Retrieved from SlideShare: https://www.slideshare.net/snoreen/mobile-cloud-computing-28973818

[29] Tobias, K. (2014). Collection Cookbook. Retrieved from KNIME Blog: http://www.knime.org/blog/collectioncookbook

[30] Vijayata, W., \& Debajyoti, M. (2014). Mobile Agent based Market Basket Analysis on Cloud. India. 2014 International Conference on IT. (pp. 49-62). Pune, India: Department Of Information Technology, Maharashtra. Institute of Technology Pune, India

[31] Woo, J. (2015). Market Basket Analysis using Spark. ARPN Journal of Science and Technology, V(4), 207-209 
APPENDICES

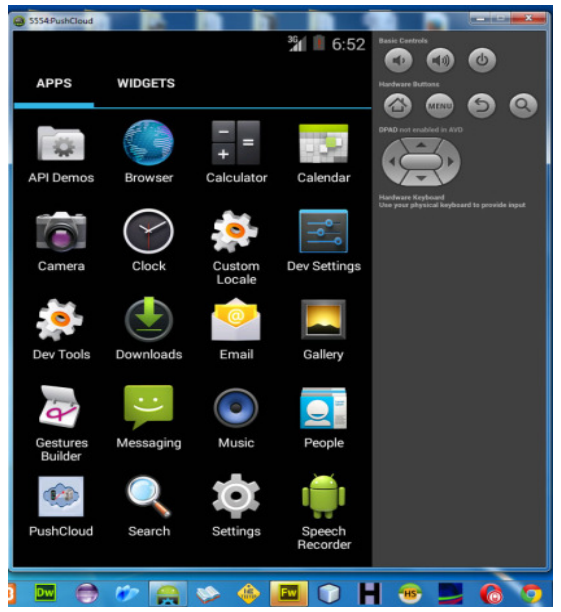

Appendix A. Snapshot of PushCloud App installed on Android.

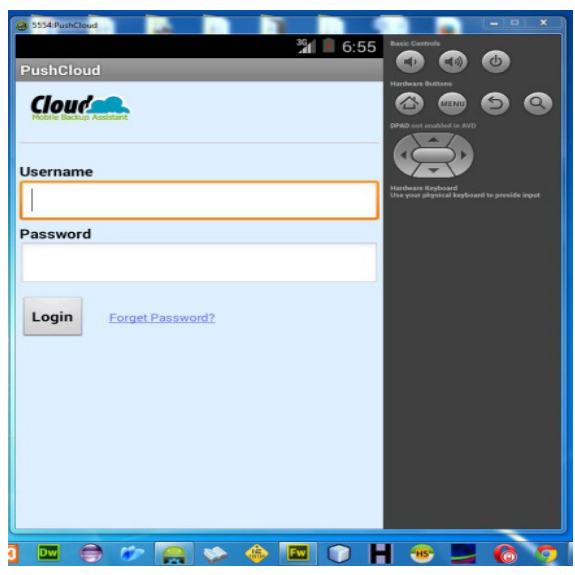

Appendix B. User Account Login on PushCloud.

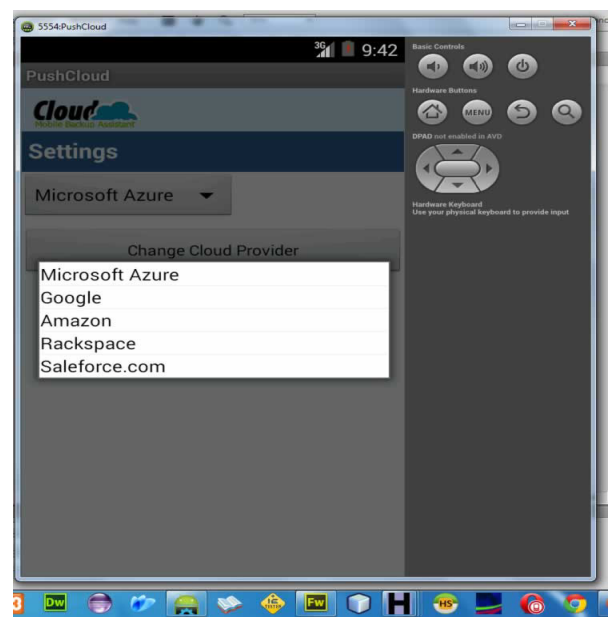

Appendix C. Documents List with Backup Option. 